\title{
New microwave power control technique by light shift detection in the DM-CPT clock
}

A. Bouvier, E. De Clercq, S. Guérendel

LNE-SYRTE, Observatoire de Paris, Université PSL, CNRS, Sorbonne Université

Paris, France

alexandre.bouvier@obspm.fr

\author{
C. E. Calosso \\ Istituto Nazionale di Ricerca \\ Metrologia \\ Turino, Italia \\ c.calosso@inrim.it
}

\author{
P. Yun \\ Key Laboratory of Time and \\ Frequency Primary Standards, \\ NTSC, Chinese Academy of \\ Sciences \\ Xi'an, China \\ yunenxue@ntsc.ac.cn
}

\begin{abstract}
Atomic clocks based on coherent population trapping (CPT) and constructive polarization modulation are promising for high performance and compact devices. We propose a new method to act on the power repartition between the two laser frequency sidebands via the microwave power. We show an improvement of the frequency stability by a factor 10 after $1000 \mathrm{~s}$ integration time. Now we are mainly limited by the temperature variations of the polarization modulator.
\end{abstract}

Keywords: Compact atomic clock, CPT, Metrology, vapor-cell, frequency standards

\section{INTRODUCTION}

Compact atomic clocks are necessary where there is a need of a precise time and frequency reference for on-boards applications. They are widely used in satellites, inertial navigation, data transfer, telecommunication synchronization, etc.

The Coherent Population Trapping (CPT) clock is a promising way to implement a high performance clock into on-board systems such as the next generation GNSS. Among the different vapor cell clocks, the Double Modulation CPT (DM-CPT) ${ }^{\text {? }}$ offers an alternative compact optical technology without any microwave cavity. We modulate synchronously the phase and the polarization of a bichromatic laser beam in order to trap more atoms in the so called dark state, improving the atomic signal. Thanks to the linear architecture (reported in figure ??), the DM-CPT clock offers interesting perspectives towards a high performance integrated device, which could be miniature with a low power consumption.

In this proceeding, we present results obtained with a new method that acts on power repartition between the two laser sidebands used for the CPT via the microwave power. We show it was one of the two main limitations of the clock frequency stability. We demonstrate a frequency stability better by a factor 10 at 1000 s integration time using this method and show the new limitation is mainly our polarization rotator.

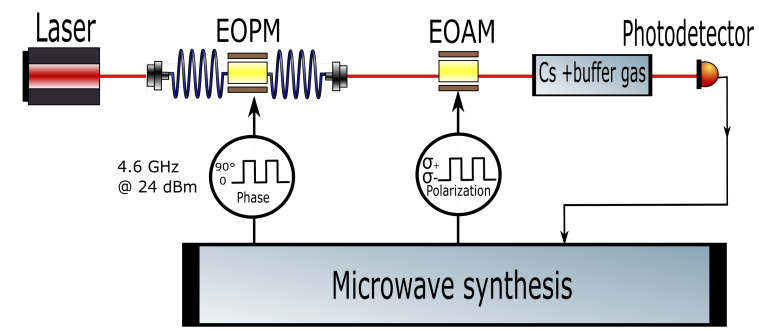

Figure 1. DM-CPT compact atomic clock simplified scheme, laser power and frequency servo-loops not represented. DFB laser source emits at $895 \mathrm{~nm}$. The microwave synthesis generates all the modulation signals and corrects the local oscillator frequency according to the detected signal after the Cs cell. The synthesis also controls the microwave power at 4.6 $\mathrm{GHz}$ transmitted to the EOPM that produces sidebands from the input laser beam. The EOAM modulates the laser polarization.

\section{CONTEXT}

Previous results has been demonstrated using the set up described in in the figure ??. It is a simple in line optical scheme. We modulate a Distributed FeedBack laser source at 4,6 GHz around the central frequency in order to generate a bichromatic laser field where each side-band is separated by $9.2 \mathrm{GHz}$ one from the other. Note this is done by feeding a $4.6 \mathrm{GHz}$ to an EOPM where the microwave power influences the optical power repartition between the sidebands. The laser source is then power and frequency locked. We then modulate synchronously the phase and the polarisation of our laser beam before the clock cell, hence the name double modulation (DM). The polarisation modulation 
keeps the atoms out of the extreme Zeeman sublevels and then let them participate more to the clock signal. The phase modulation allows the constructive superposition of the CPT dark states. ? Therefore, the DM scheme allows us to have a good contrast of the atomic transition we use to lock the oscillator on.

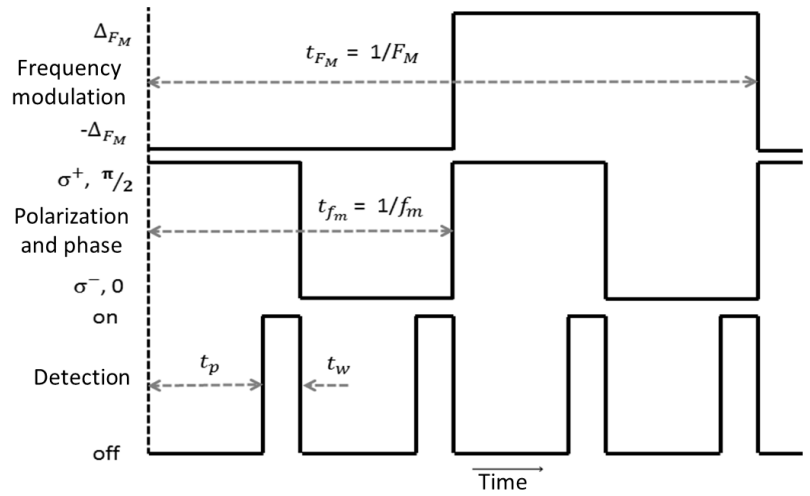

Figure 2. Time sequence used. Frequency is modulated at $F_{m}=250 \mathrm{~Hz}$ at $\Delta_{F_{M}}=130 \mathrm{~Hz}$ depth. Pumping time and detection window are noted respectively $t_{p}$ and $t_{w}$.

The polarisation and phase are modulated synchr-onously according to the time sequence depicted in figure ?? and said previously. At each phase/polarisation, after a time $t_{p}$ where the atoms are pumped in the CPT state, we detect the signal during a time $t_{w}$.

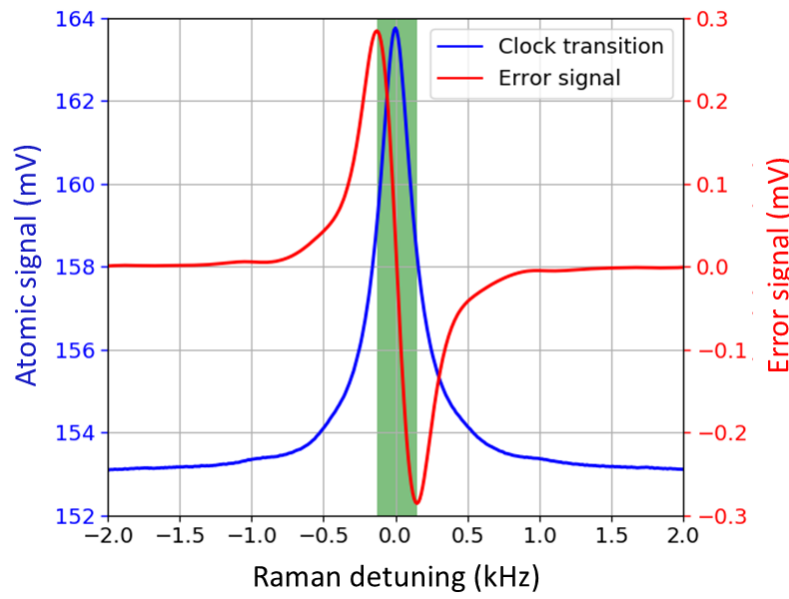

Figure 3. Atomic signal (blue) and error signal (red), we obtained a contrast of $6.98 \%$ for a full width at half maximum of $290 \mathrm{~Hz}$ represented in green.

The error signal used to lock the clock frequency is generated by square modulating the microwave frequency at a frequency of $F_{m}=250 \mathrm{~Hz}$ with a depth of $\Delta_{F_{M}}=130 \mathrm{~Hz}$ around the clock frequency. The obtained signal is also shown in the figure ??, it presents a contrast of $6.98 \%$ for a $290 \mathrm{~Hz}$ at full width at half maximum.

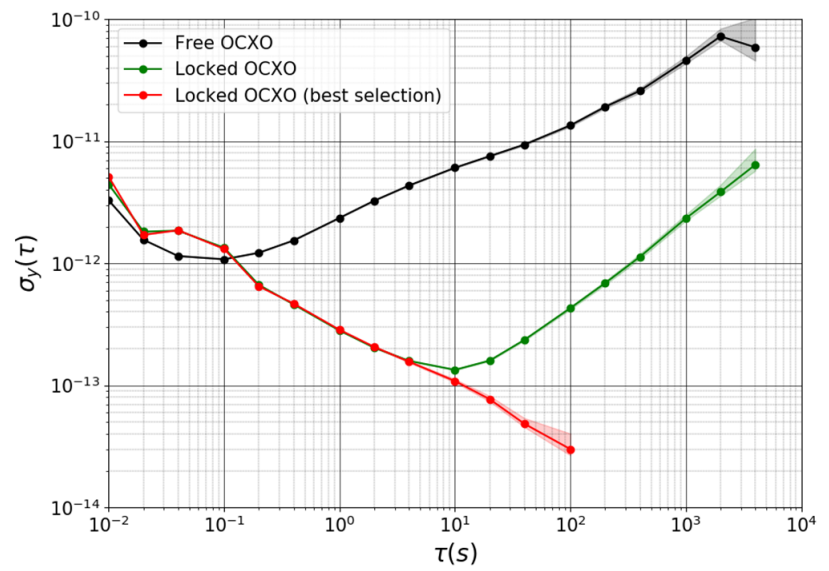

Figure 4. Frequency stability of the DM-CPT atomic clock. We measured a stability of $2.9 \times 10^{-13}$ up to $100 \mathrm{~s}$. At 1000 $\mathrm{s}$ integration time we are limited to $2.1 \times 10^{-12}$.

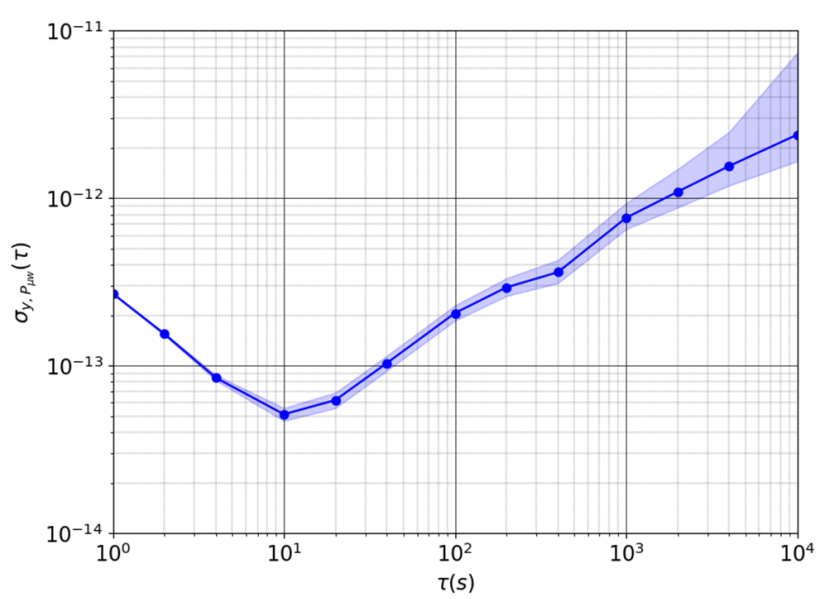

Figure 5. Contribution of the microwave power to the clock frequency stability measured at a level of $7.6 \times 10^{-13}$.

Frequency stability of $2.9 \times 10^{-13}$ at $1 \mathrm{~s}$ up to $100 \mathrm{~s}$ and $2.1 \times 10^{-12}$ at $1000 \mathrm{~s}$ has been measured with this set-up as reported in figure ??. Here, we will only describe the two main contribitions since they are at least 10 time more important than the others. We also will limit to the stability at $1000 \mathrm{~s}$ since the frequency stability at that integration time was our main objective. First contribution we will adress is the microwave power. It is responsible for the optical power repartition between the sidebands used for the CPT. Instabilities of the microwave power induced then inbalance between those sidebands and the carrier. Measuring a sensibility of clock frequency of $-7.9 \mathrm{~Hz} \cdot \mathrm{dB}^{-1}$, we can calculate the contribution reported in the figure ?? based on the 
microwave power stability. We have then a contribution of $7.6 \times 10^{-13}$. Note that at this point, the microwave power is not regulated.

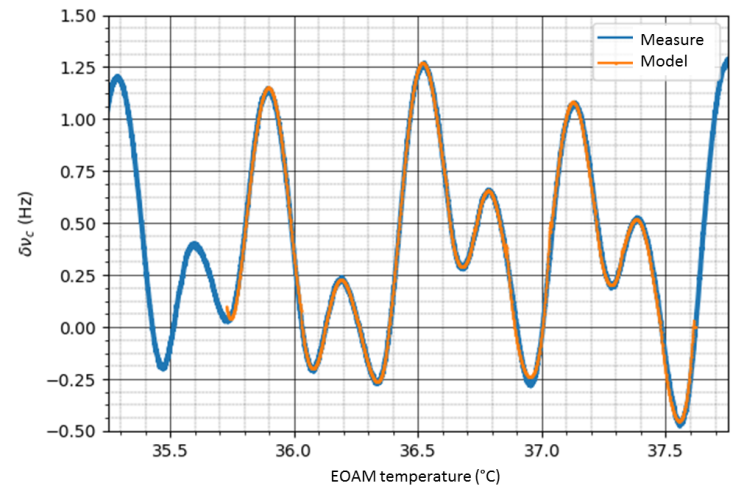

Figure 6. Clock frequency as a function of the temperature of the amplitude modulator modulator (in blue) and a model allowing to convert the temperature fluctuations of the EOAM in clock frequency fluctuations (in orange).

The other main contribution was the temperature of the polarization rotator (EOAM). In facts, we have observed the phenomenon despicted in the figure ??. We attribute this behaviour to the intern reflexions happening in the EOAM. The latter is composed of two triangular crystals placed in a staggered pattern a few millimeters apart. Despite our effort, we could not get rid of this effect, so we have decided to regulate its temperature on a maximum. In order to estimate its contribution to the clock frequency stability, we have modeled this behaviour with a polynomial set. Therefore we can now estimate its contribution based on a temperature measurement and we have determined a contribution of $6.9 \times 10^{-13}$.

\section{MICROWAVE POWER CONTROL}

This method has been inspired by a collaboration between Femto-ST, INRIM and SYRTE.?, Our idea is to use a particularity of the DM-CPT clock in order to control the laser power repartition between the sidebands via the microwave power. As shown in figure ??, the frequency shift induced by a total laser power variation depends also of the microwave. Therefore, by modulating the total optical power before the clock cell we can measure the light shift and then generate an error signal which can be used to correct the microwave power. In other words, we lock the microwave power on the atomic signal. This methods differs from the one recently published? because it acts directly on the cause of the light shift.

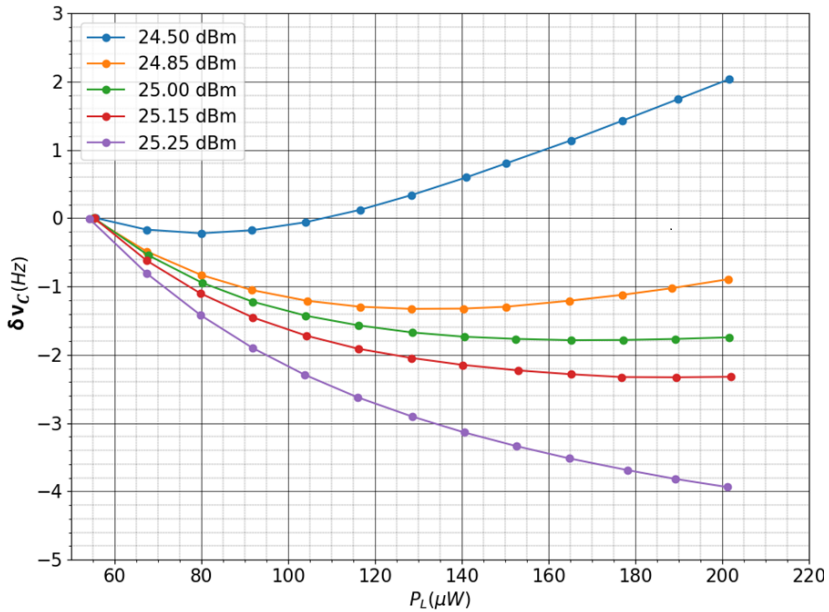

Figure 7. DM-CPT clock frequency shift as function of the laser power and the microwave power.

The new time sequence used is given in figure ?? It is globaly the same as the one in figure ??, exept we modulate the laser power. At each of them, we modulate the modulate the phase and the polarisation as previously explained. We also apply a symmetry in the interrogation in order to get rid of a memory effect of the atoms described in. ? The determination of the working parameters has been detailled in.? We chose those in a way that we can have the same error signal slope for the two laser power used with the same light induced shift. We also took care of choosing points where the stability at $1 \mathrm{~s}$ integration would not be too degraded.

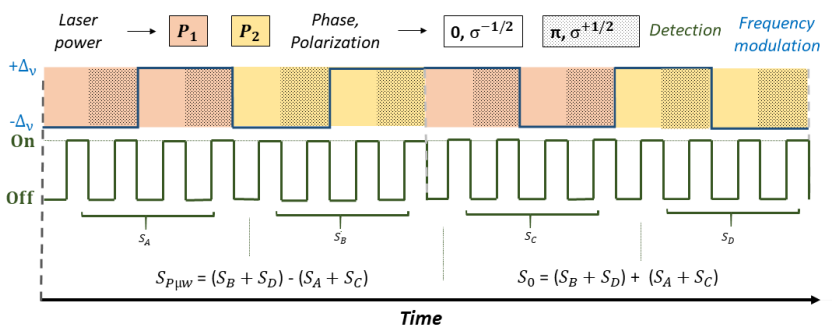

Figure 8. Time sequence used to lock the local oscillator while correcting the microwave power. In pink and yellow the two laser powers we are working with. In grey we see the phase and polarisation modulations of the laser beam. The blue line represents the frequency modulation of the local oscillator and the green one the detection periods. Below are the summations of the signals, $S_{P u w}$ is the signal used for the microwave power control and $S_{0}$ is the signal used to control the oscillator.

\section{RESULTS AND NEW LIMITATIONS}

The frequency stability obtained with this method is reported in figure ??. We can see a slight degradation 
at $1 \mathrm{~s}$ compared to previous results, from to $2.9 \times 10^{-13}$ to $3.3 \times 10^{-13}$. Despite this, the stability at $1 \mathrm{~s}$ is still acceptable compared to other compact atomic clocks. At $1000 \mathrm{~s}$ integration time, we observe a stability of $2.1 \times 10^{-13}$, better by a factor 10 compared to the previous results, proving the efficiency of this new method.

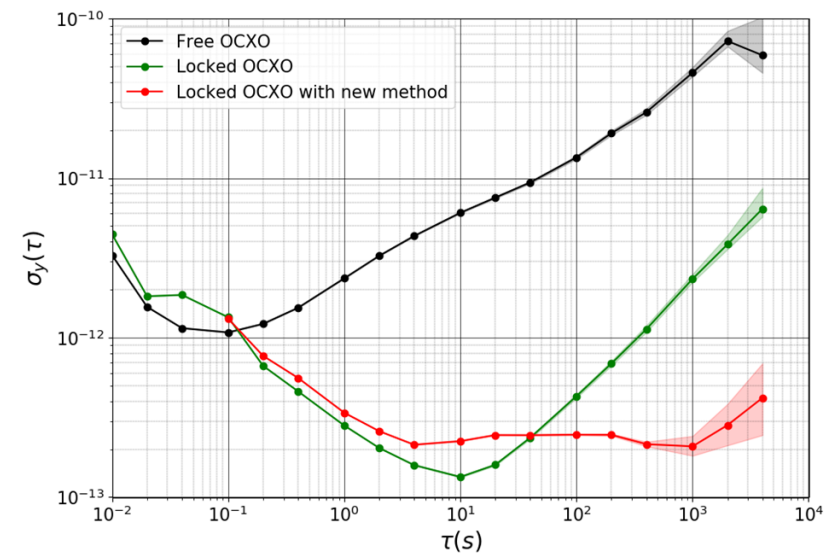

Figure 9. Clock frequency stability. In black the free OCXO, in green the OCXO locked with the previous method and in red locked with the new one.

A more in-depth study has been conducted in order to estimate the new levels of the two previous main contributions. We first measured the microwave power stability as reported in figure ??. We observe a stability better by a factor 8 at $1000 \mathrm{~s}$. With a new sensibility of the clock frequency of $-10.9 \mathrm{~Hz} \cdot \mathrm{dB}^{-1}$ we obtain a contribution of $1.6 \times 10^{-13}$.

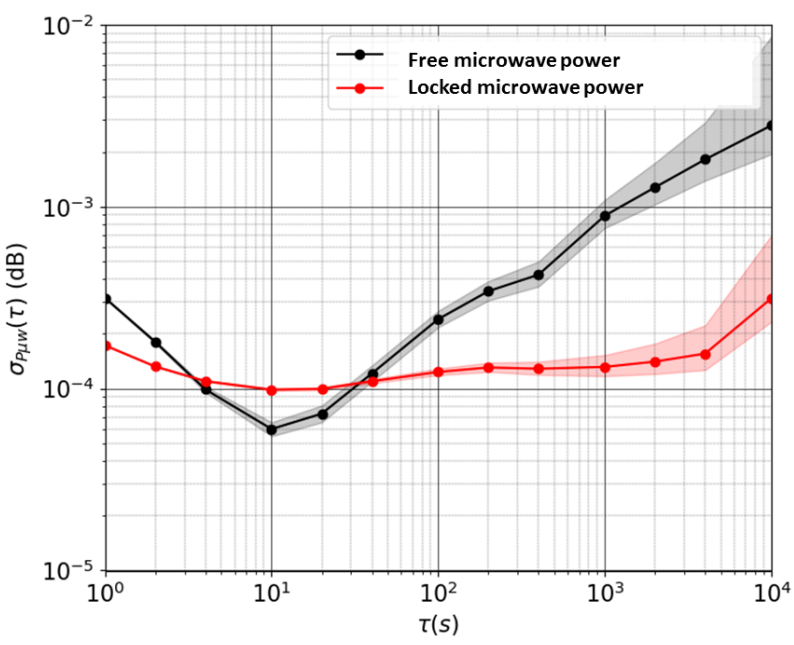

Figure 10. Microwave power stability. We observe a gain of a factor 8 at $1000 \mathrm{~s}$ with the new method.

Secondly, we measured the stability of the polariza- tion modulator (EOAM) temperature in order to calculate its contribution to the clock frequency stability. We did this by the mean of a polynomial set. This contribution is reported in the figure ??. We measured a contribution of $1.8 \times 10^{-13}$, which is better by a factor 3. We explain this behaviour by the fact that the impact of the EOAM is now correctd by the mean of the microwave power.

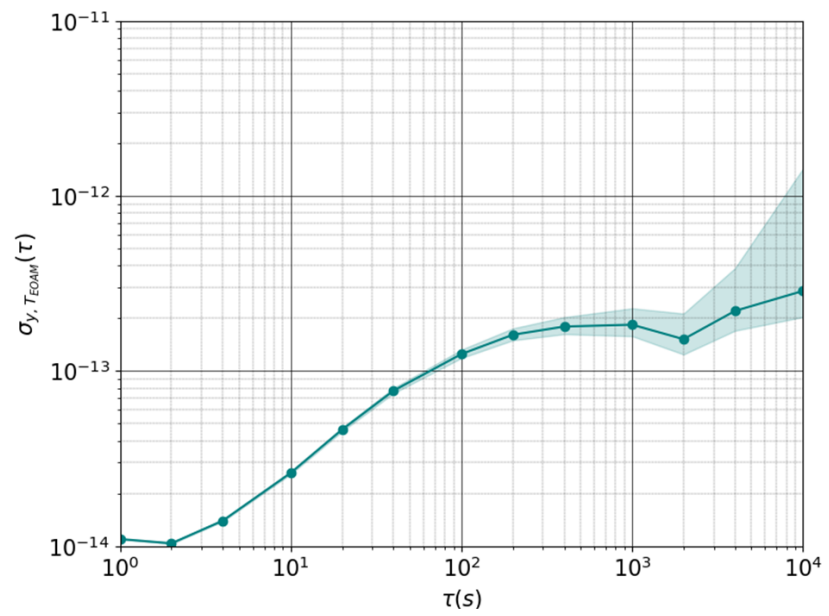

Figure 11. Polarization modulator (EOAM) temperature contribution to the clock frequency stability measured at $1.8 \times 10^{-13}$ after $1000 \mathrm{~s}$ integration time.

The polarization modulator being already temperature regulated, we will not be able to reduce its contribution as it is now. However, we plan to remplace it with an other technology that should not present the same behaviour, reducing its contribution to the frequency stability.

\section{CONCLUSION}

We presented the results obtained with a new method controlling the optical power repartition in the sidebands generated by an EOPM by the mean of the microwave power. A stability better by a factor 10 has been measured at $1000 \mathrm{~s}$ integration time, proving the efficiency of this method. However, the two main limitations to the frequency stability remain the microwave power and the temperature of the polarization modulator. The first being now locked on the atomic signal, we should achieve a better microwave stability by reducing the contribution of the temperature of the polarization modulator. This can be achieve by changing the technology used to rotate the polarization. Thus, it should also allow a better clock frequency stability. However, studies about the dependencies between the two main 
limitations should be conducted in order to confirm this assomption.

\section{ACKNOWLEDGMENTS}

We want to the electronic department at the SYRTE for their time and precious advices and the European Space Agency for the loan of the Symmetricom 5125a. We also want to thank FIRST-TF for the co-funding of the $\mathrm{PhD}$ thesis and the CNES for having followed this project.

\section{REFERENCES}

1. M. Langlois, L. De Sarlo, D. Holleville, N. Dimarcq, J.-F. Schaff, and S. Bernon, "Compact coldatom clock for onboard timebase: tests in reduced gravity," Physical Review Applied 10(6), p. 064007, 2018.

2. P. Yun, F. Tricot, C. E. Calosso, S. Micalizio, B. Francois, R. Boudot, S. Guerandel, and E. De Clercq, "High-performance coherent population trapping clock with polarization modulation," Physical Review Applied 7(1), p. 014018, 2017.

3. P. Yun, S. Guérandel, and E. De Clercq, "Coherent population trapping with polarization modulation," Journal of Applied Physics 119(24), p. 244502, 2016.

4. C. Calosso, B. Francois, R. Boudot, P. Yun, M. Gozzelino, E. Bertacco, and S. Micalizio, "Local oscillators and digital electronics for compact atomic clocks," in Microwave Technology and Technique Workshop, 2017.

5. C. Calosso, M. Gozzelino, H. Lin, F. Levi, A. Godone, and S. Micalizio, "Novel techniques for locking the laser frequency to the clock cell in vapor cell standards," in Proc. 2019 Intern. Freq. Contr. Symp., Paper WeBT2, 1, 2019.

6. M. A. Hafiz, R. Vicarini, N. Passilly, C. Calosso, V. Maurice, J. Pollock, A. Taichenachev, V. Yudin, J. Kitching, and R. Boudot, "Advanced light-shift compensation protocol in a continuous-wave microcell atomic clock," arXiv preprint arXiv:2005.05096 , 2020.

7. A. Bouvier, C. Calosso, P. Yun, E. de Clercq, and S. Guerandel, "Studies on the mid-term effects of the double-modulation cpt clock," in International Conference on Space Optics-ICSO 2020, 11852, p. 1185247, International Society for Optics and Photonics, 2021. 